\title{
Reflection on the Components of University Identity System
}

\author{
Zhao Yuhong ${ }^{1, a}$, Dao Zhuoda ${ }^{2,3, b^{*}}$ \\ ${ }^{1}$ Xishuangbanna Vocational and Technical College, Xishuangbanna, Yunnan, China \\ ${ }^{2}$ Xishuangbanna Media Group, Xishuangbanna, Yunnan, China \\ ${ }^{3}$ College of Humanities, Yantai University, Yantai, Shandong, China \\ ayaojiayj70@126.com \\ b*daozhuoda@foxmail.com
}

\begin{abstract}
Reviewing the existing theoretical framework of the University Identity System (UIS), this paper reorganizes the components of UIS by combing related theories and practical experience, proposes three subsystems as the components of UIS, scilicet, Cultural Identity System (CIS), Behavioral Identity System (BIS), and Presentational Identity System (PIS), and explains the concepts and relationships of the three subsystems.
\end{abstract}

Keywords: University Identity System, Cultural Identity System, Presentational Identity System

\section{高校识别系统构成的再思考}

\author{
赵育红 ${ }^{1, a}$ 万卓达 ${ }^{2,3, b *}$
}

${ }^{1}$ 西双版纳职业技术学院，西双版纳，云南，中国

${ }^{2}$ 西双版纳广播电视台, 西双版纳, 云南, 中国

3烟台大学人文学院, 烟台, 山东, 中国

a yaojiayj70@126. com

b* daozhuoda@foxmail. com

\section{摘要}

本文通过回顾高校识别系统（UIS）已有的理论框架，结合相关理论与实践经验对 UIS 的构成进行再梳理，据 此提出 UIS 构成的三个子系统, 即文化识别系统 (CIS)、行为识别系统 (BIS) 与呈现识别系统 (PIS), 并解 释了三个子系统之概念及其相互关系。

关键词：高校识别系统，文化识别系统，呈现识别系统

\section{1. 引言}

随着我国高等教育理论与实践经验的不断积累, 高等学校的治理层次愈加立体。作为企业识别系统 (Corporate Identity System) 在高校的在地化呈现, 高校识别系统 (University Identity System, UIS) 的相 关理论与研究也愈加凸显出中国本土特色。UIS 沿用 企业识别系统的理论框架, 将理念识别系统 (Mind Identity System, MIS)、行为识别系统 (Behavioral
Identity System, BIS) 和视觉识别系统 (Visual Identity System, VIS）三个子系统作为其基本构成及研究路 径。[1]49-50 在对 UIS 研究不断深入的同时, 一些相关 概念也被作为 UIS 的其他构成相继提出，如表 1 所 示。这些概念虽为更加全面地呈现 UIS 的图景提供了 可能, 但因缺乏严格和清晰的界定，导致这些概念在 具体的意指上出现混乱, 使得 UIS 难以形成系统性的 研究框架和具有可行性的操作方法。 


\section{表 1 UIS 子系统的相关概念}

\begin{tabular}{|l|c|}
\hline \multicolumn{1}{|c|}{ 中 } & 艾 \\
\hline 理念识别系统 & Mind Identity System \\
\hline 行为识别系统 & Behavioral Identity System \\
\hline 视觉识别系统 & Visual Identity System \\
\hline 文化识别系统 & Cultural Identity System \\
\hline 发展识别系统 & Developmental Identity System \\
\hline 听觉识别系统 & Audio Identity System \\
\hline 嗅觉识别系统 & Olfactory Identity System \\
\hline 环境识别系统 & Environmental Identity System \\
\hline 资料来源: $\mathrm{Lu}^{[2] ; ~ L a i ~}{ }^{[3] ; ~ Z h a n g ~}{ }^{[4]}$ 等 \\
\hline
\end{tabular}

本文基于 UIS 的既有理论, 结合建构中所积累的 实践经验, 从构成要素及其相互关系上对有关子系统 的概念进行梳理、辨析和界定，以期提出一种更为系 统化的 UIS 构成, 进而为 UIS 的研究与实践提供更 为科学的理念和路径。

\section{2. 从 MIS 到 CIS 的转变}

UIS 语境下的 MIS，是指高校的教育理念、价值 取向和办学宗旨等在精神层面上用以区分外者的规 范系统。 ${ }^{[2]}$ 以山东大学为例, 其 MIS 就包含办学宗旨、 办学目标、校训、校风等内容。Zhang 指出, MIS 是 高校形象的根本与灵魂, BIS 和 VIS 是 MIS 的反映 和具体化。 ${ }^{[5]}$ MIS 与 BIS、VIS 之关系正如一个人的 内在需借助其行为与外貌方能体现一样。

在对 UIS 的一般认知中, 文化识别系统 (Cultural Identity System, CIS) 和发展识别系统 (Developmental Identity System, DIS）分别强调的是高校在已有文化 和发展历程上区别于其他高校的特质, 关注的是以往 的状态和积累。然而, UIS 却更多是指向未来, 要求 具有先进性与前瞻性的, 这就使得各子系统在时间上 存在着矛盾关系。实际上, MIS、CIS 与 DIS 都是精 神层面的概念, 而 CIS 的外延更大, 包含了精神层面 上的其他子系统。因此, 无论以时间为标准亦或是以 其他为标准, 都没有必要再去进行二次划分。在实践 中, 直接就可以用 CIS 涵盖和代替其他精神层面上的 子系统。

Melewar 在回顾企业形象（corporate identity）的 构成时, 提出企业形象包含如图 1 所示的几个子系统 和具体概念。 ${ }^{[6]}$

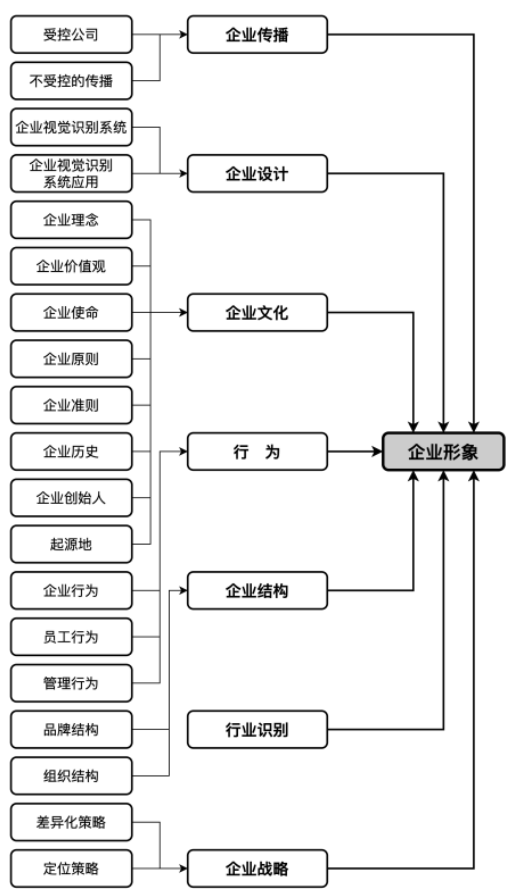

图 1 企业形象的构成

Melewar 将企业理念 (corporate philosophy)、企 业价值观（corporate values）、企业准则（corporate principles) 等类 MIS 的概念, 与企业使命 (corporate mission）和企业历史（corporate history）一同归入企 业文化（corporate culture）的范畴中。正如 Albert 所 主张, 组织的文化反映着这一组织的核心价值观、行 为与信念。 ${ }^{[7]}$ Sun 也赞成将校园文化视为高校形象的 构成要素之一, 认为校园文化是学校理念与精神的传 承载体。 ${ }^{[8]}$ 因此, 不妨将 UIS 中的理念用意义更广的 文化所替代, 即以 CIS 取代 MIS。于此, 作为 UIS 子 系统的 CIS, 通过借助 BIS 和 VIS 的实施, 使高校得 以在精神层面与其他高校达成区分和识别。

\section{3. 从 VIS 到 PIS 的转变}

VIS 是借助视觉形象达成高校识别的系统, 包括 学校名称标准字体、校徽、印章等构成的基础系统和 办公事务用品、车体外观、服饰规范等构成的应用系 统两部分。 ${ }^{\left[{ }^{9}\right]}$ VIS 将学校的办学理念、文化特质等非 视觉语言转换为具体符号、图形等概念, 以有形的方 式展示出来, 通过有序组织、统一风格、定制标准、 规范系统的视觉方案体现学校的办学方针和文化内 涵, 从而形成特有的学校形象。 ${ }^{[10]}$ 也就是说, 高校借 助 VIS 这一介质将自身理念传达给师生员工或公众, 进而完成高校的形象定位，如图 2 所示。

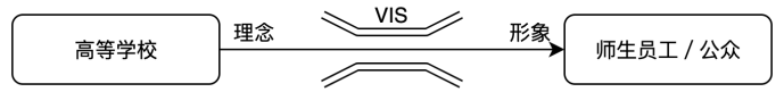

图 2 VIS 的运行机制

听觉识别系统 (Audio Identity System, AIS) 和 嗅觉识别系统 (Olfactory Identity System, OIS) 分别 
指透过听觉和嗅觉来展现高校形象。AIS 在高校中最 显著的体现即是校歌, 而 OIS 作为由气味营销延伸出 的一种新概念，也被逐渐运用到高校形象的建构之中。 ${ }^{[11]} \mathrm{AIS}$ 及 OIS 在建构高校形象的过程与机制上与 VIS 并无差异，只是在具体呈现方式上有所差异，如表 2 所示。

表 2 AIS、0IS 及 VIS 的差异

\begin{tabular}{|c|c|c|c|}
\hline & AIS & OIS & \multicolumn{2}{|c|}{ VIS } \\
\hline 涉及感观 & 听 觉 & 嗅 觉 & 视 觉 \\
\hline
\end{tabular}

环境识别系统 (Environmental Identity System, EIS ) 则是以校园环境为识别依据的系统。EIS 与 VIS 同样都是通过视觉呈现高校形象的系统，而前者的视 觉符号载体一般是静态的建筑与景观, 后者则一般是 动态的物件。 $\mathrm{Li}^{[1] 52} 、 \mathrm{Pan}^{[12]} 、 \mathrm{Zhu}^{[13]}$ 等人也把包括建筑 和景观在内的校园环境看作是一体化的符号表现形 式, 将其归入 VIS 的范畴中, 进而发挥其结构筑建的 功能。

可见, AIS、OIS、EIS 及 VIS 是同一范畴下依据 所涉及感官和传达载体之不同而划分出的概念。因此, 完全可以将 VIS、AIS、OIS 及 EIS 归于同一范畴, 正 如 Baumeister 所言, “呈现是一种向他人传递自身形 象的一种行为” [14], 将其看作一种高校的自我呈现, 统称作呈现识别系统 (Presentational Identity System, PIS)。

\section{4. 关于 BIS 的再思考}

BIS 是指在理念精神的指导下形成的一系列活动, 它相当于一个人的手脚, 涉及到组织行为运作的全方 位和全过程。[15]关于 MIS、BIS 及 VIS 三者间的关系, 有学者认为, MIS 决定 BIS 进而决定 VIS, 如图 3 所 示。[16][17][18]

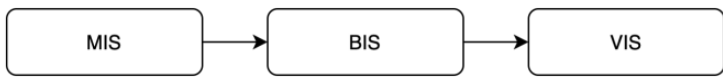

图 3 关于 MIS、BIS、VIS 三者关系的一种假说

然而, 这种假说是站不住脚的。UIS 理论认为, 高校标志是最核心的 VIS 要素, ${ }^{[19]}$ 而在实践中, 很多 没有 BIS 的学校却仍有校徽。这一论断到底是以 BIS 的外在称名而存在, 还是以其规范内容而存在, 尚待 商榷。如果 BIS 是以规范内容的存在而存在的话, 那 就是说, 校园内的所有行为都属于 BIS 的范畴, 就会 将其他的子系统淹没，如图 4 所示。但在实践当中， 更多的事例证明, 各个子系统是独立存在的, 如北京 大学的《北京大学视觉形象识别系统管理办法》。所 以, 以规范内容作为 BIS 存在的依据, 在逻辑上是无 法自洽的。

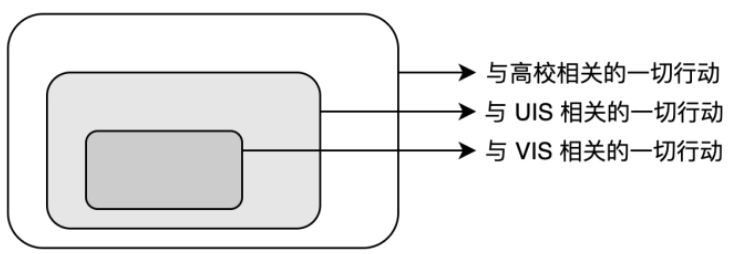

图 4 BIS 中行动的不同界定

正如 Chen 所指出, UIS 由以 MIS 为基础的理论 元素和以平面印刷设计、环境识别、数码媒体、行为 识别等其他识别应用的符号元素共同构成。[20]在以往 的 UIS 理论中, BIS 的概念涵盖了 VIS 的范畴, 这种 扩张使得两者之界限模糊不清, 理念间的平衡也被打 破。因此, 本文认为, BIS 的定义应当仅限于其外在 称名的范畴, 不宜扩大解释, 即 BIS 仅指人的外在行 为表现或外在行为呈现的系统。在建构 BIS 时, 应当 严格遵守这一界限, 如此才能使 CIS、BIS、PIS 各司 其职, 发挥作用, 如图 5 所示。

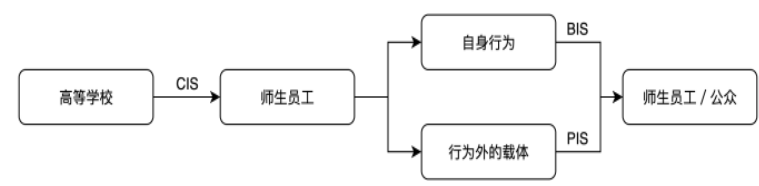

图 5 CIS、BIS 及 PIS 的基本关系

\section{5. 结论}

基于以上的论述，本文认为， UIS 的构成应当由 CIS 及以 CIS 为主导的 BIS 和 PIS 构成, 如图 6 所 示。其中, CIS 是代表高校文化、理念等精神层面、 意识层面的系统，是整个系统的核心部分，决定了其 他两个子系统的性质和程度。BIS 是以人的行为呈现 高校形象, PIS 是以其他的外在载体呈现高校形象, 两者作为形式符号共同承载着作为内涵意义的 CIS, 从而构成完整的、有活力的、有价值的 UIS。

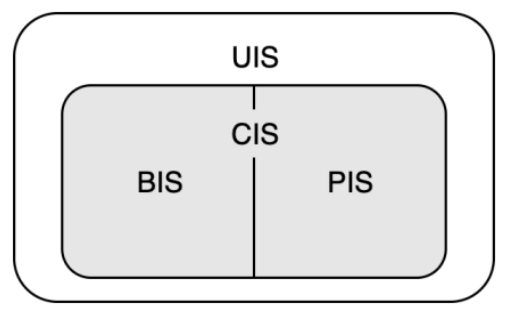

图 6 UIS 的构成

通过对 UIS 子系统的梳理和辨析, 便可引申出 UIS 的运行机制。高校借由 CIS、BIS 和 PIS 规范师 生员工的行为, 师生员工借助自身行为赋予 UIS 内涵 与价值, 同时结合行为外的其他载体促进和完善 UIS 的构建与提升, 为高校的形象建设与呈现提供更多的 手段与途径。

建设高水平大学、培养高素质人才, 是提升我国 文化竞争力和增强综合国力的必然要求。构建 UIS, 不是为了区别而区别，不是为了识别而识别，而是通 
过外在的 UIS，促使高校形成、加深、完善自己独特 的精神文明和校园文化。通过建构完整的 UIS, 使得 各子系统相对独立又相互配合, 夯实校园文化底蕴, 提升师生员工的道德水平、职业素养及治学精神，提 升高校的综合实力, 进而从本质上拥有区别于其他高 校的特质, 并通过 UIS 识别出来。与此同时, 这种内 部的运转又能促使这一外在系统的形式设置得更为 科学、合理, 并且为提升这一系统的建设提供新的理 论与方法, 形成良性循环, 达到构建 UIS 的初衷与目 的。

\section{REFERENCES}

[1] Li, J. (2012) University Identity System in Campus Brand Building. Modern Education Management, 6.

[2] Lu, L., Zhang, F.W. (2011) On Characteristics of Visual Identity System. Journal of Zhejiang Business Technology Institute, 10(2): 37.

[3] Lai, Y., Liu, Y.G. (2005) Discussion on the Construction of University Identifying System. Higher Education Forum, 5: 172.

[4] Zhang, Q. (2013) On University Image Identity System - A Case Study of Lanzhou University. Journal of Lanzhou University (Social Sciences), 41(3): 158-162.

[5] Zhang, H.Q. (2002) On Building the Image of the University and the Publicizing of the Image. Journal of Hunan Mass Media Vocational and Technical College, 2: 79.

[6] Melewar, T.C., Karaosmanoglu, E., Paterson, D. (2005) Corporate Identity: Concept, Components and Contribution. Journal of General Management, 31(1): 62 .

[7] Albert, S., Whetten, D.A. (1985) Organizational identity. Research in Organizational Behavior, 7: 263-295.

[8] Sun, K.S. (2013) The Action of Campus Identity System to Enhance the Image of Universities. Higher Education Forum, 9: 124.

[9] Gao, L.J. (2011) Design and Transmission of Visual Image Identifying System in College Images. Journal of Luoyang Institute of Science and Technology (Social Science Edition), 26(5): 91.
[10] Yang, H.Y., Zhao, L., Liu Y.N. (2015) A Study on the Connotation of the Visual Image Recognition System. Journal of North China Institute of Aerospace Engineering, 25(4): 1.

[11] Ouyang, Y. (2014) Research on University Visual Identity System Based on Brand and Culture. (MA thesis). Hefei University of Technology: 3334.

[12] Pan, Y., Han, M. (2017) The Importance of the Design and Development of Visual Identity System in the Construction of Green Campus: in Lanzhou University of Finance Longqiao College as an Example. Design Community, S1: 93-94.

[13] Zhu, J., Chen, Y. (2018) Study on Design Strategy for Campus Visual Identity and Space Design in University. Architecture \& Culture, 6: 202-203.

[14] Baumeister, R.F., Hutton, D.G. (1987) SelfPresentation Theory: Self-Construction and Audience Pleasing. In: Mullen, B., Goethals, G.R. (Eds.), Theories of Group Behavior. Springer, New York. p. 71.

[15] Liu, Y. (2000) Using CI Strategy for Reference in Developing Institutions of Higher Learning. Journal of Lanzhou Jiaotong University, 2: 84.

[16] Liu, D. (2006) Corporation Identity System. Journal of Northeast Agricultural University (Social Science Edition), 1: 28.

[17] Ge, J.J., Song, D.H. (2003) An Introduction to the Designing of University Identify System (UIS). Journal of Guilin University of Electronic Technology, 2: 68-69.

[18] Luo, J. (2009) CI-System - Means of Building the Images of Higher Education Institutes. Journal of Xinjiang Normal University (Edition of Philosophy and Social Sciences), 30(2): 143.

[19] Hong, Q. (2013) Research on the Design Strategy of Chinese Contemporary University Logo. The Science Education Article Collects, 3: 134.

[20] Chen, L., Zhou, H.H. (2005) On the Construction and Management of University Identity. Journal of Nanjing University of Aeronautics and Astronautics (Social Sciences), 3: 32. 